\title{
Zika virus pandemic: a human and public health crisis
}

\author{
Guilherme Sousa Ribeiro ${ }^{[1],[2]}$ and Uriel Kitron ${ }^{[1],[3]}$
}

[1]. Centro de Pesquisas Gonçalo Moniz, Fundação Oswaldo Cruz, Salvador, Bahia, Brasil. [2]. Instituto de Saúde Coletiva, Universidade Federal da Bahia, Salvador, Bahia, Brasil. [3]. Department of Environmental Sciences, Emory University, Atlanta, United States of America.

\section{Zika virus spread}

Late in 2014, clusters of cases of an acute exanthematous illness were first noticed in several municipalities of Northeastern Brazil. Within months, case numbers exploded throughout the region, and local outpatient units overflowed with patients complaining of pruritic rash, accompanied in less than half of cases by low-grade fever, arthralgia, headaches, and myalgia. The initial suspected etiology of the outbreaks was dengue fever or chikungunya. However, Brazil has experienced large dengue epidemics since the 1990s, and clinical manifestations were atypical for dengue, with patients rarely exhibiting high-grade fever. The mild arthralgia also made chikungunya, introduced into Brazil in 2014, less likely. The etiological diagnosis remained a mystery until April 2015, when, almost simultaneously, researchers identified the Zika virus in serum samples from patients in Bahia ${ }^{(1)}$ and Rio Grande do Norte ${ }^{(2)}$, two Northeastern states $\sim 1,000 \mathrm{~km}$ apart.

By late 2015, autochthonous Zika cases were laboratory confirmed in 19 of Brazil's 27 federative units, but accurate case number estimates are unavailable, as Zika was not a mandatory reportable disease. In Salvador, Bahia's capital, the local epidemiological surveillance office established a notification system for the so-called indeterminate acute exanthematous illness, and from February to June 2015, about 15,000 cases were reported ${ }^{(3)}$, representing an attack rate of 5.5 cases $/ 1,000$ persons. However, empirical observation suggests that the proportion of clinically affected was much greater, possibly reaching one-third of the city's population.

Zika virus was not the sole arbovirus driving patients to Brazil's health units in 2015. Laboratory investigation of exanthematous outbreak cases from Salvador also confirmed dengue and chikungunya among the reported $\operatorname{cases}^{(3)}$. Additionally, according to the Brazilian Ministry of Health, 1.65 million suspected dengue cases were reported in 2015, the highest annual number ever reported in Brazil, making misdiagnoses between Zika and dengue probable.

Zika is an ribonucleic acid (RNA) flavivirus transmitted to humans by Aedes mosquitoes. It was first isolated from

Corresponding author: Dr. Guilherme S. Ribeiro.

e-mail: guilherme.ribeiro@bahia.fiocruz.br

Received 28 January 2016

Accepted 15 February 2016 monkeys in the Zika forest, Uganda, in 1947 (Figure 1). Few human cases were reported until the first known outbreak in Yap Island, Micronesia, in $2007^{(4)}$. Additional outbreaks occurred in French Polynesia in $2013^{(5)}$ and Cook Island and New Caledonia in 2014 ${ }^{(6)}$. The first autochthonous circulation of Zika virus in the Americas was detected in a Chilean Pacific Island, between February and June 2014(7). However, only following the introduction of Zika into Brazil have outbreaks spread throughout Latin America and the Caribbean. As of mid-January 2016, autochthonous Zika virus circulation has been detected in 18 countries and territories in the Americas ${ }^{(7)}$, and 27 worldwide ${ }^{(8)}$.

\section{Zika virus infection complications and public health impact}

During the 2013 French Polynesia outbreak, GuillainBarré syndrome (GBS), a neurological disease clinically characterized by progressive and ascendant muscle weakness, increased in occurrence ${ }^{(8)}$. An increase in GBS cases was also identified soon after detection of Zika virus circulation in Northeastern Brazil, El Salvador, and Venezuela ${ }^{(8)}$. Other autoimmune and neurological syndromes, such as meningitis, meningoencephalitis, and myelitis, may also be associated with Zika virus infection and merit further investigation.

The game changer was the declaration by the Brazilian government in October 2015 of a national public health emergency due to a drastic increase in children born with microcephaly in the State of Pernambuco. Microcephaly is a congenital syndrome involving abnormal brain development and subsequent smaller-than-normal head circumference. Prenatal Zika virus infection is the suspected cause of microcephaly and thus may become the first great plague of the $21^{\text {st }}$ century.

In just 3 months between initiation of microcephaly monitoring (22 October, 2015) and the Ministry of Health public update of 20 January, 2016, 3,893 suspected cases of microcephaly were reported in Brazil, from 21 of the 27 federative units and 764 municipalities. Three Northeast states account for the vast majority of reported cases (Pernambuco: 1,306 cases; Paraíba: 665 cases; and Bahia: 496 cases). Among 512 clinically investigated cases, the microcephaly diagnosis was confirmed in 230. Data from French Polynesia also suggests an increase in central nervous system malformations in newborns and fetuses during 2014-2015(8). 


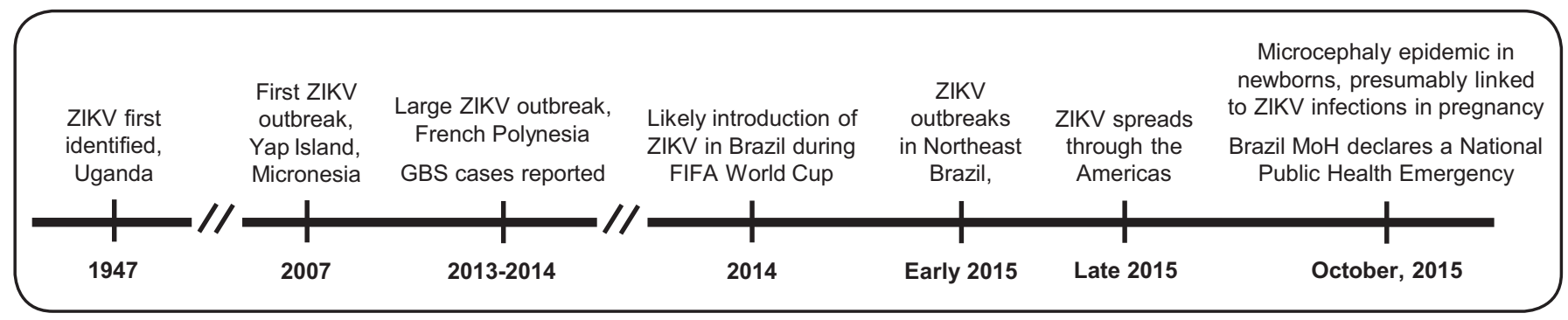

FIGURE 1 - Epidemiological timeline of Zika virus global spread. ZIKV: Zika virus. GBS: Guillain-Barré syndrome; FIFA: Fédération Internationale de Football Association; MoH: Ministry of Health.

Other neurological problems, such as hypertonia/spasticity, hyperreflexia, and seizures have been identified in children born with microcephaly ${ }^{(9)}$. However, there remains an urgent need to follow pregnant women diagnosed with Zika infection to assess the risk of abortions, microcephaly, and other milder neurological malformations of their infants. Studies to determine the long-term prognosis of afflicted babies are also needed. Although affected children are still $<6$ months old, the prognosis for neurological recovery is minimal, given that $\sim 70 \%$ of them presented severe microcephaly ${ }^{(9)}$.

The spatiotemporal correlation of Zika virus epidemics with an observed increase in GBS cases soon after, and in the frequency of children born with microcephaly months later suggest an association. However, definitive laboratory and epidemiological studies to establish a causal relationship are still pending. As of January 2016, laboratory evidence for an etiological role of prenatal Zika virus infection in the occurrence of microcephaly remained scarce $^{(7)}$. Zika virus RNA was detected in the amniotic fluid of two fetuses with microcephaly according to prenatal ultrasound, and in the placenta of a woman who manifested Zika infection in early pregnancy and aborted. In addition, the virus was detected by reverse transcriptionpolymerase chain reaction (RT-PCR) and immunohistochemistry in four cases of congenital malformation: two miscarriages and two full-term newborns who died soon following delivery. RTPCR confirmation of Zika virus infection in children born with microcephaly has been challenging, underscoring the need for accurate serological tests.

As the Zika virus epidemics in the Northeast likely peaked between April and June 2015, we predict the number of microcephaly cases in the region to spike in the coming weeks and then decline. However, Zika virus transmission continues throughout other regions of Brazil and has spread to other tropical and subtropical countries. If the link between prenatal Zika virus infection and microcephaly is confirmed, we will observe a further worldwide rise in newborns with microcephaly and other congenital impairments. Therefore, there is an urgent need for health services to adapt to provide assistance for this large population of affected children, who will require neuroimaging and neurological diagnosis, and longterm support from neurologists, physiotherapists, occupational therapists, and other health professionals.

\section{Scientific and infrastructure gaps for Zika virus control and prevention}

Various questions remain unanswered. Why did the Zika virus spread so quickly in Brazil and from Brazil to the rest of the Americas? Did the virus mutate, resulting in increased vector competence? Do other potential routes of virus transmission have any epidemiological significance? What are the attack rate and basic reproduction number $\left(\mathrm{R}_{0}\right)$ for Zika? Does the previous observation that about $80 \%$ of infections were asymptomatic in French Polynesia hold for Brazil and the rest of the Americas? What percentage of the population in different regions of Brazil did the virus infect? Does an infection provide longterm immunity, as believed? If so, will herd immunity prevent extensive Zika virus transmission in regions where large outbreaks have occurred? Does prior exposure to dengue play a role? Will endemic transmission continue? What is the risk of congenital malformations for babies whose mother was infected during pregnancy? Is microcephaly the tip of the iceberg for other milder congenital syndromes? If so, which ones and how often?

In addition to these scientific knowledge gaps, the abrupt emergence of Zika caught public health services unprepared to address the huge disease-associated burden. Currently, serological methods for diagnosing Zika virus are hampered by cross-reactivity with other flaviviruses, especially dengue. Only a few laboratories in Brazil and in other countries have the capacity to diagnose Zika virus infection by RT-PCR. Consequently, the number of laboratory-confirmed Zika cases in Brazil remains extremely low, given that the Ministry of Health estimates that between 500,000 and 1.5 million people were infected with Zika in 2015.

Current methods for Aedes control in Brazil have failed, as evidenced by repeated dengue epidemics and the introduction and spread of Zika and chikungunya viruses ${ }^{(10)}$. As an affordable vaccine against dengue, Zika, and chikungunya viruses may not be available for years, evaluation of alternative vector control methods, e.g., biological larva control and use of Wolbachiainfected or transgenic mosquitoes, and improved application of traditional methods are warranted. Furthermore, major investments in urban infrastructure, water supply, sanitation, garbage collection, and housing are essential. Without these infrastructure improvements, no specific method targeting mosquito populations or arbovirus transmission will be effective. 


\section{Conclusions}

This tragic scenario, with a generation of children born with neurological disorders that may impose severe lifelong limitations, requires the public, public health professionals, and governments to find effective responses to mitigate the current crisis and prevent recurrence. The upcoming Olympic Games later this year, with thousands of visitors at risk of exposure, may accelerate the spread of Zika and other arboviruses worldwide, and only adds to the need for urgent measures and long-term plans.

\section{ACKNOWLEDGMENTS}

The authors thank the staff of the Secretary of Health of Salvador, Bahia for their collaborative support.

\section{CONFLICT OF INTEREST}

The authors declare that there is no conflict of interest.

\section{FINANCIAL SUPPORT}

Brazilian National Council for Scientific and Technological Development (CNPq) for a grant (400830/2013-2).

\section{REFERENCES}

1. Campos GS, Bandeira AC, Sardi SI. Zika virus outbreak, Bahia, Brazil. Emerg Infect Dis 2015; 21:1885-1886.
2. Zanluca C, De Melo VC, Mosimann AL, Dos Santos GI, Dos Santos CN, Luz K. First report of autochthonous transmission of Zika virus in Brazil. Mem Inst Oswaldo Cruz 2015; 110:569-572.

3. Cardoso CW, Paploski IAD, Kikuti M, Rodrigues MS, Silva MMO, Campos GS, et al. Outbreak of exanthematous illness associated with Zika, chikungunya, and dengue viruses, Salvador, Brazil. Emerg Infect Dis 2015; 21:2274-2276.

4. Duffy MR, Chen T-H, Hancock WT, Powers AM, Kool JL, Lanciotti RS, et al. Zika virus outbreak on Yap Island, Federated States of Micronesia. N Engl J Med 2009; 360:2536-2543.

5. Cao-Lormeau V-M, Roche C, Teissier A, Robin E, Berry AL, Mallet HP, et al. Zika virus, French Polynesia, South Pacific, 2013. Emerg Infect Dis 2014; 20:1085-1086.

6. Roth A, Mercier A, Lepers C, Hoy D, Duituturaga S, Benyon E, et al. Concurrent outbreaks of dengue, chikungunya and Zika virus infections - an unprecedented epidemic wave of mosquitoborne viruses in the Pacific, 2012-2014. Euro Surveill 2014; 19:pii=20929.

7. Pan American Health Organization/World Health Organization. Epidemiological Update: Neurological syndrome, congenital anomalies and Zika virus infection, 17 January 2016. Washington, DC: PAHO/WHO; 2016.

8. European Centre for Disease Prevention and Control. Zika virus epidemic in the Americas: potential association with microcephaly and Guillain-Barré syndrome (first update), 21 January 2016. Stockholm: ECDC; 2016.

9. Schuler-Faccini L, Ribeiro EM, Feitosa IML, Horovitz DDG, Cavalcanti DP, Pessoa A, et al. Possible association between Zika virus infection and microcephaly - Brazil, 2015. MMWR Morb Mortal Wkly Rep 2016; 65:1-4.

10. Marcondes CB, Ximenes MFFM. Zika virus in Brazil and the danger of infestation by Aedes (Stegomyia) mosquitoes. Rev Soc Bras Med Trop 2016; 49:4-10. 